\title{
Coherent Oscillations inside a Quantum Manifold Stabilized by Dissipation
}

\author{
S. Touzard, ${ }^{1, *}$ A. Grimm, ${ }^{1}$ Z. Leghtas, ${ }^{1}$ S. O. Mundhada, ${ }^{1}$ P. Reinhold, ${ }_{1}^{1}$ C. Axline, ${ }^{1}$ M. Reagor, ${ }^{1}$ K. Chou, ${ }^{1}$ \\ J. Blumoff, ${ }^{1}$ K. M. Sliwa, ${ }^{1}$ S. Shankar, ${ }^{1}$ L. Frunzio, ${ }^{1}$ R. J. Schoelkopf, ${ }^{1}$ M. Mirrahimi, ${ }^{2}$ and M. H. Devoret ${ }^{1}$ \\ ${ }^{1}$ Department of Applied Physics and Physics, Yale University, New Haven, Connecticut 06520, USA \\ ${ }^{2}$ Yale Quantum Institute, Yale University, New Haven, Connecticut 06520, USA \\ and QUANTIC team, INRIA de Paris, 2 Rue Simone Iff, 75012 Paris, France
}

(Received 15 November 2017; revised manuscript received 19 January 2018; published 4 April 2018)

\begin{abstract}
Manipulating the state of a logical quantum bit (qubit) usually comes at the expense of exposing it to decoherence. Fault-tolerant quantum computing tackles this problem by manipulating quantum information within a stable manifold of a larger Hilbert space, whose symmetries restrict the number of independent errors. The remaining errors do not affect the quantum computation and are correctable after the fact. Here we implement the autonomous stabilization of an encoding manifold spanned by Schrödinger cat states in a superconducting cavity. We show Zeno-driven coherent oscillations between these states analogous to the Rabi rotation of a qubit protected against phase flips. Such gates are compatible with quantum error correction and hence are crucial for fault-tolerant logical qubits.
\end{abstract}

DOI: 10.1103/PhysRevX.8.021005

The quantum Zeno effect (QZE) is the apparent freezing of a quantum system in one state under the influence of a continuous observation. This continuous observation can be performed by a dissipative environment [1-3]. It can be further generalized to the stabilization of a manifold spanned by multiple quantum states, an operation which requires a dissipation that is blind to the manifold observables [4]. Harnessing this effect is crucial for the design of quantum computation schemes, since autonomous stabilization is a form of the feedback needed for quantum error correction. When employing manifold QZE for correcting errors, motion inside the manifold can still subsist and can be driven by the combination of the dissipative stabilization and an external force [5-10]. Therefore, manifold QZE offers a pathway towards the realization of logical gates compatible with quantum error correction. An example of such a system is provided by a superconducting microwave cavity, in which a dissipative process that annihilates photons in pairs at rate $\kappa_{2}$, acting together with a twophoton drive of strength $\epsilon_{2}$, projects the system onto the manifold spanned by Schrödinger cat states $\left|\mathcal{C}_{\alpha_{\infty}}^{ \pm}\right\rangle=$ $\mathcal{N}\left(\left|\alpha_{\infty}\right\rangle \pm\left|-\alpha_{\infty}\right\rangle\right)$, where $\left|\alpha_{\infty}\right\rangle$ is a coherent state of amplitude $\alpha_{\infty}=\sqrt{2 \epsilon_{2} / \kappa_{2}}$ and $\mathcal{N}$ is a normalization factor [11-13]. Each one of these states has a well-defined photon number parity, which is conserved by the engineered

\footnotetext{
*steven.touzard@yale.edu
}

Published by the American Physical Society under the terms of the Creative Commons Attribution 4.0 International license. Further distribution of this work must maintain attribution to the author(s) and the published article's title, journal citation, and DOI.
Subject Areas: Quantum Physics, Quantum Information

dissipation. In this Schrödinger cat state manifold, the displacement operator $\mathbf{D}(\alpha)=\exp \left(\alpha \mathbf{a}^{\dagger}-\alpha^{*} \mathbf{a}\right)$ (where $\mathbf{a}$ is the annihilation operator acting on the harmonic oscillator) has two effects: it changes the photon number parity and it changes the amplitude of its component coherent states. The engineered dissipation leaves the change in parity invariant and cancels the change in amplitude [Fig. 1(a)]. The net result of this quantum Zeno dynamics is to continuously vary the parity of Schrödinger cat states.

These parity oscillations constitute the basis of an $X$ gate on a qubit encoded in the protected manifold $|0 / 1\rangle_{P}=$ $\mathcal{N}\left(\left|\alpha_{\infty}\right\rangle \pm\left|-\alpha_{\infty}\right\rangle\right)$. Encoding quantum information in superpositions of Schrödinger cat states is compatible with quantum error correction realized with quantum nondemolition parity measurements [14-16]. Our gate is fundamentally different than previous manipulations of Schrödinger cat states [17] as it operates while the manifold is stabilized. Thus, the quantum information is protected from out-ofmanifold gate errors. Moreover, the operation of the gate is not affected by the dominant source of errors: bit flips. In fact, as the operation commutes with them, it is compatible with a fault-tolerant scheme that would correct them after the operation.

While related driven manifold dynamics have been proposed and observed [18-22], the nonlinear dissipation specific to our experiment adds a crucial element: any drift out of the cat state manifold is projected back into it.

In our experiment, schematically shown in Fig. 1, the drive dissipation is implemented using two-photon transitions between two electromagnetic modes. The first one has a high quality factor and stores the Schrödinger cat states. We refer to it as the storage (subscript $S$ ). The second one is used as an engineered cold bath that rapidly removes 


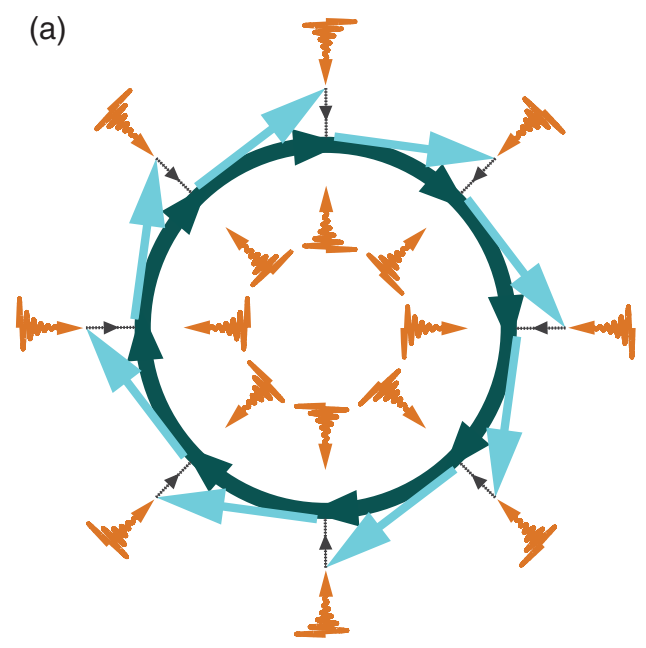

(b)

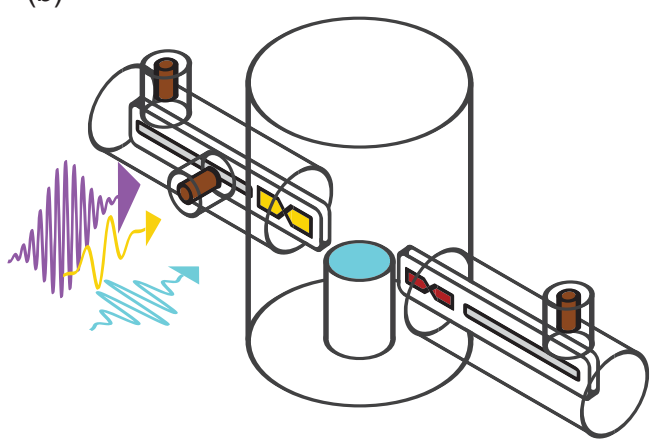

FIG. 1. Quantum Zeno dynamics and its implementation. (a) Conceptual representation of the experiment. The quantum state of a harmonic oscillator is represented here by a point in a 2D plane (not to be confused with phase space). The dark blue circle represents a cross section of the Bloch sphere of a two-state manifold in the larger Hilbert space of the oscillator. The quantum Zeno effect observed in our experiment corresponds to motion along the circle. A weak excitation drive is applied to the oscillator and the resulting trajectory has a component both along the circle and out of it. The nonlinear dissipation and drive (orange) cancels the movement outside the circle while being blind to the position of the quantum state on the circle. (b) Schematics of the experimental device. The quantum manifold is stabilized within the Hilbert space of the fundamental mode of an aluminium post cavity (cyan, storage in the text). This resonator is coupled to two Josephson junctions on sapphire (yellow for the reservoir and crimson for the Wigner transmon, see text), which are read out by stripline resonators (gray). Three couplers (brown) bring microwave drives into the system and carry signals out of it.

the entropy from the storage. We refer to it as the reservoir (subscript $R$ ). We employ the four-wave mixing capability of a Josephson junction, together with two microwave pumps, to stimulate those transitions. In order to make resonant the conversion from one reservoir photon into two storage photons and vice versa, the first pump is set at frequency $2 f_{S}-f_{R}$. The second pump, set at frequency $f_{R}$, combines with the first one to create pairs of photons in the storage. When the dynamics of the reservoir mode is eliminated, the density matrix of the storage mode $\rho$ is given by the Lindblad equation,

$$
\frac{d \rho}{d t}=-\frac{i}{\hbar}\left[\mathbf{H}_{S}, \rho\right]+\frac{\kappa_{2}}{2} \mathcal{D}\left[\mathbf{a}_{S}^{2}-\alpha_{\infty}^{2}\right] \rho,
$$

where $\mathbf{H}_{S}$ is a Hamiltonian acting on the storage and $\mathcal{D}[\mathbf{L}] \rho=2 \mathbf{L} \rho \mathbf{L}^{\dagger}-\mathbf{L}^{\dagger} \mathbf{L} \rho-\rho \mathbf{L}^{\dagger} \mathbf{L}$ is the Lindblad superoperator. As the Lindblad superoperator is engineered to be the dominant term in the dynamics, the dynamical steady states of the system are given by the coherent states $\left| \pm \alpha_{\infty}\right\rangle$. The microwave pumps set the phase and amplitude of the complex amplitude $\alpha_{\infty}$. The Hamiltonian part of the equation contains the self-Kerr effect of the storage mode induced by the Josephson junction and the linear drive that induces the coherent oscillations: $\mathbf{H}_{S} / \hbar=$ $-\chi_{S S} / 2\left(\mathbf{a}_{S}^{\dagger 2} \mathbf{a}_{S}^{2}\right)+\left(\epsilon \mathbf{a}_{S}^{\dagger}+\right.$ H.c. $)$. The frequency of the coherent oscillations is maximum when the phase of the linear drive $\epsilon$ is perpendicular to the phase of the stabilized Schrödinger cat states. Thus, this linear drive displaces the Schrödinger cat state perpendicularly to the stabilization axis while the dissipation continuously projects the system back to the stabilized manifold. If the drive respects the adiabaticity condition $|\epsilon| \ll\left|\alpha_{\infty}\right|^{2} \kappa_{2}$. then the net effect of the linear drive is to induce parity oscillations within the stabilized manifold, at frequency $\Omega=2 \epsilon\left|\alpha_{\infty}\right|$ [12].

The adiabaticity condition [6-10] sets an upper bound on the frequency of these oscillations, fixed by the maximum $\kappa_{2}$ that we can engineer. In order to observe this dynamics, we also need the coherence time of the storage mode to be larger than the period of the oscillations. The architecture we designed was key to engineer both a highly coherent storage mode and a large coupling to the environment. We implement the storage mode into a long-lived post cavity made of aluminium [23] [Fig. 1(b)]. Its finite lifetime induces two types of errors on a protected qubit encoded in the stabilized manifold. First, in the absence of stabilization, the amplitude of the Schrödinger cat states decays until eventually the two coherent states are no longer distinguishable. This error happens at rate $\kappa_{1}^{-1}=92 \mu \mathrm{s}$. Second, when the environment is observed to have absorbed a photon, the projected density matrix of the storage mode suffers a parity jump, which corresponds to a bit-flip error in our encoded qubit. For a stabilized cat state containing $\bar{n}=\left|\alpha_{\infty}\right|^{2}$ photons on average, they happen at a rate $\bar{n} \kappa_{1}$ [24]. Additionally, the abovementioned Kerr effect would distort the coherent states at rate $\chi_{S S} / 2 \pi \sim 3 \mathrm{kHz}$ in the absence of stabilization. In order to achieve a fast nonlinear dissipation, the storage cavity is coupled to a transmon embedded into a coaxial tunnel [25], whose lifetime is engineered to be much less than that of the storage (317 ns), and we use it as the entropy reservoir to induce the QZE. While the reservoir is efficient to dispose of the entropy of the storage mode, its low lifetime has two impacts on the coherence of the storage. First, we associate 
the lifetime of our storage cavity to the Purcell effect (usually $\kappa_{1}^{-1}$ is of order $1 \mathrm{~ms}$ [23]). Second, the finite temperature of the reservoir causes additional dephasing of the storage mode (see Supplemental Material [26]). However, the direct coupling between the storage and the reservoir modes leads to a nonlinear dissipation rate of $\kappa_{2}^{-1}=900 \mathrm{~ns}$. The 2 orders of magnitude separating $\kappa_{1}$ and $\kappa_{2}$ are enough to observe the parity oscillations while respecting the adiabaticity condition. The storage cavity is also coupled to a very coherent transmon whose coherence times $\left(T_{1}=70 \mu \mathrm{s}, T_{2}^{*}=30 \mu \mathrm{s}\right)$ are large compared to the time it takes to perform a parity measurement of the storage cavity using the dispersive coupling (218 ns). We use this transmon to measure the Wigner function of the storage mode and therefore we refer to it as the Wigner transmon.

Our experimental protocol follows a fixed sequence of pulses which contains three parts [Fig. 2(a)]. The first step is the initialization of the system in the encoding manifold, which is done with pulses generated by an optimal control algorithm referred as optimal control pulse (OCP) [17]. As it involves transient states that are not Schrödinger cat states and that are entangled to the Wigner transmon, this method induces errors on the protected encoding that are not corrected by the stabilization. However, it is currently

(a)

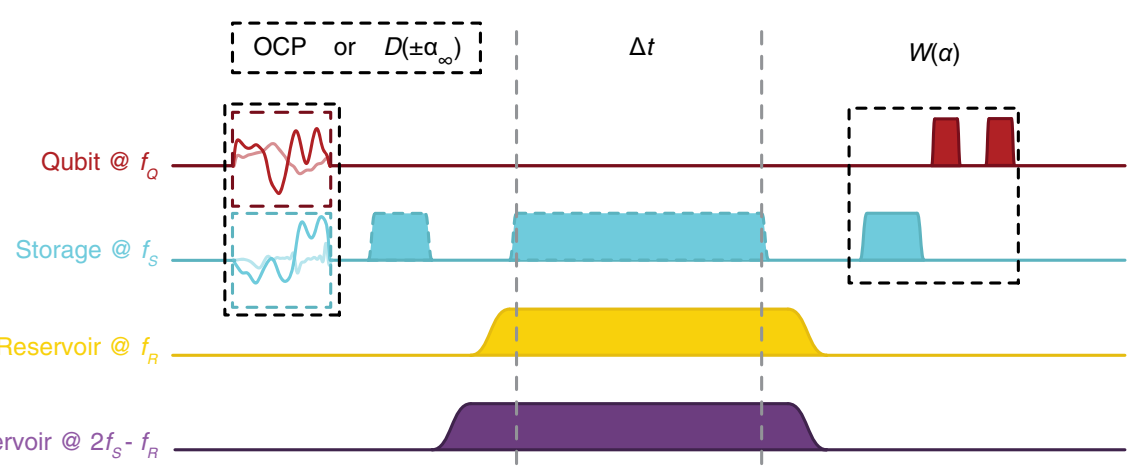

(b)

Reservoir @2 $2 f_{S}-f_{B}$
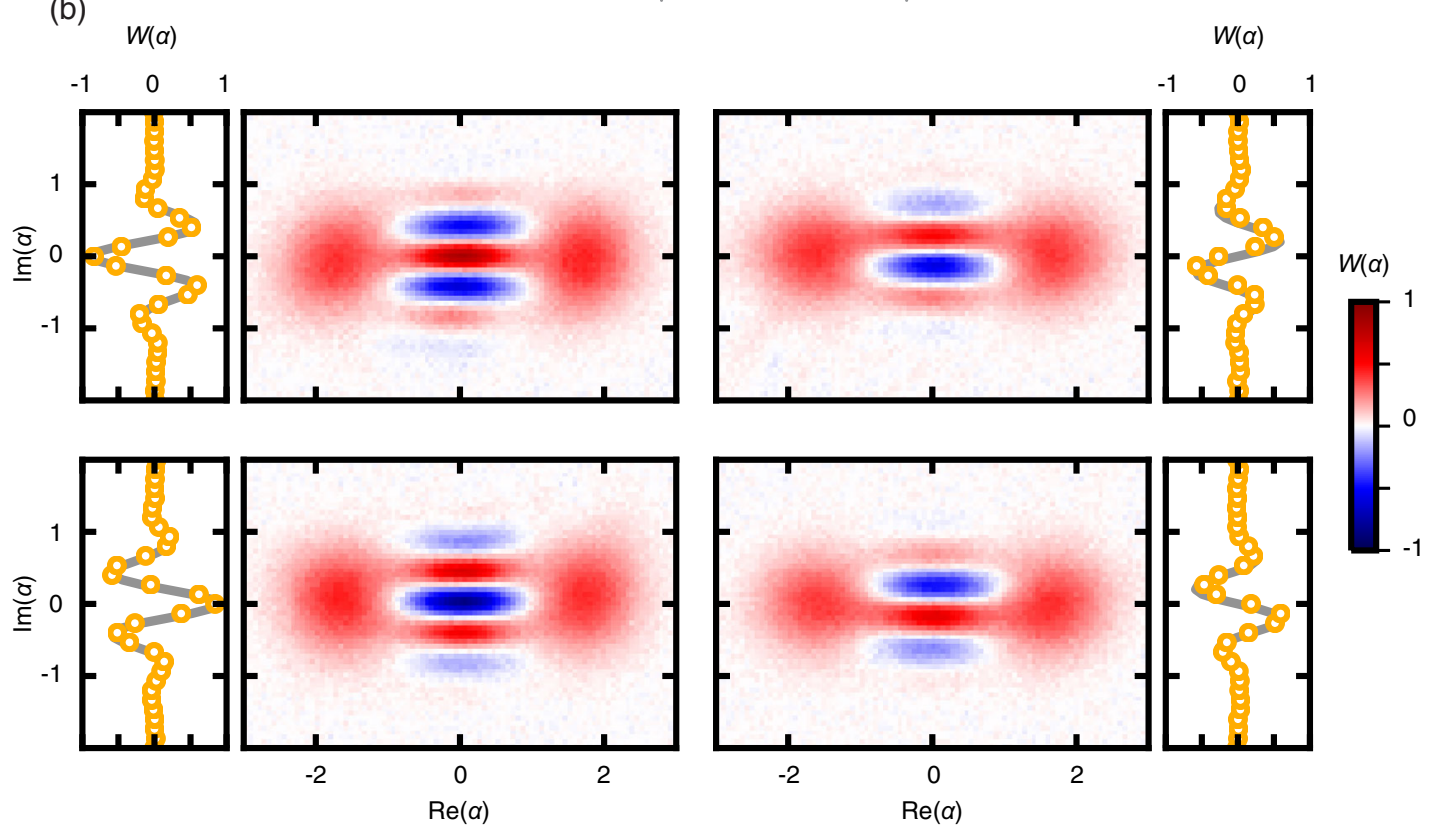

FIG. 2. Experimental protocol and Wigner tomography result. (a) Sequence of different drives outlined in Fig. 1. The storage is initialized either in a cat state $\mathcal{N}\left(\left|\alpha_{\infty}\right\rangle \pm\left|-\alpha_{\infty}\right\rangle\right)$ with optimal control pulses or in a coherent state $\left| \pm \alpha_{\infty}\right\rangle$ with a displacement $D\left( \pm \alpha_{\infty}\right)$. The drives stabilizing the manifold are turned on in $24 \mathrm{~ns}$ (purple and yellow). They are on for a duration $\Delta t$ during which the storage drive (cyan) can be turned on to induce the parity oscillations. The drives are left on for another $500 \mathrm{~ns}$ and then turned off in $24 \mathrm{~ns}$, after which the Wigner function is measured. (b) In the left-hand column is the Wigner functions of the storage cavity after initialization in an even or odd cat state $\left(\left|\alpha_{\infty}\right|^{2}=3\right)$. The right-hand column shows the corresponding Wigner functions after a quarter of an oscillation. The color maps are averaged raw data of the Wigner function measurement (see text) and the orange circles are cuts along $\operatorname{Re}(\alpha)=0$. The gray solid lines are theoretical curves corresponding to even or odd cats (left-hand column, lines 1 and 2, respectively) and parityless cats (right-hand column, lines 1 and 2). The only fit parameter in the theory is the renormalization of the amplitude by a factor 0.87 on the left, and 0.65 on the right. These factors account for the fidelity of the parity measurement and the decay of the fringes of the cat states during the stabilization. 
the fastest method available. The second part is the stabilization of the manifold, which is done with or without the rotation drive. Finally, in the third part, the Wigner function of the storage cavity at a given point in phase space is measured [14,27].

We characterize the initialization and the quality of the measurement by taking a full Wigner tomography of the storage cavity initialized in $|0\rangle_{P}$ and $|1\rangle_{P}$ [Fig. 2(b)]. The raw data consist of single-shot parity measurements realized with a parametric amplifier and averaged without any further normalization. The phase locking of the different drives ensured that the stabilization axis of the Schrödinger cat states was aligned with the Wigner representation axis, and that the rotation drive was perpendicular to the stabilization axis [26]. The right-hand column illustrates our ability to go from an even or odd parity Schrödinger cat state to a "Yurke-Stoler" cat state [28]. The zero value of the Wigner function at the center of phase space shows that these states had no parity. They were generated by a rotation of $\pi / 2$ in the encoding manifold. It is important to note that the cat is not displaced sideways. The fringes are moving, but the "blobs" remain in place [Fig. 2(b)].

In order to investigate the parity oscillations more closely, we restrict the measurement of the Wigner function to the center of phase space (photon number parity measurement). In Fig. 3(a), we present the time evolution of cat states, initially in the even state. We measured their parity over $50 \mu \mathrm{s}$ while they were stabilized [Fig. 3(a)]. For $\bar{n}=2,3$, and 5, we observed decay time constants of, respectively, 22, 14, and $8 \mu \mathrm{s}$. This behavior arises from the natural single-photon jumps of the cavity. They correspond to bit flips within the encoding manifold which eventually destroy the coherence of the encoded qubit. The coherence of the encoded qubit is lost at a rate $2 \bar{n} \kappa_{1}$ [24]. This is close to what was found in the experiment and thus shows that the decoherence is mainly due to bit flips happening during the stabilization. With the rotation drive turned on, the oscillations of the parity over time are similar to Rabi oscillations for a two-level system. For a drive with strength $\epsilon$, the equivalent Rabi frequency [12] is given by (a)
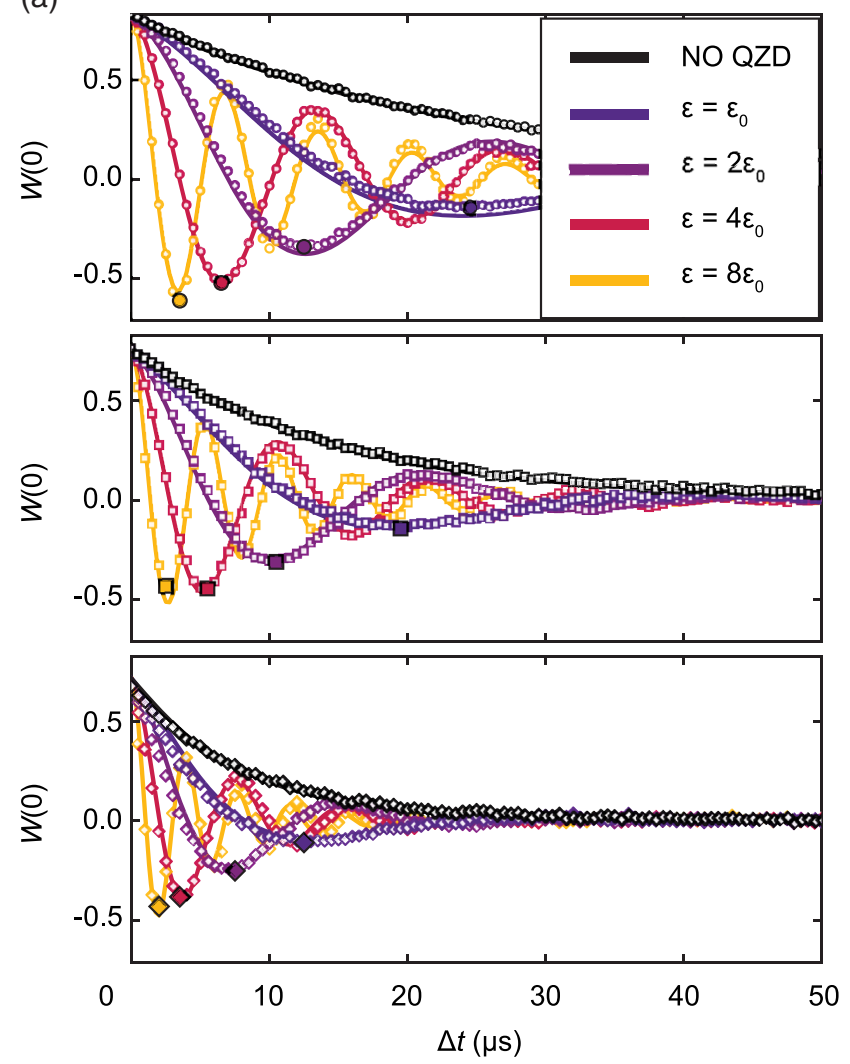

(b)

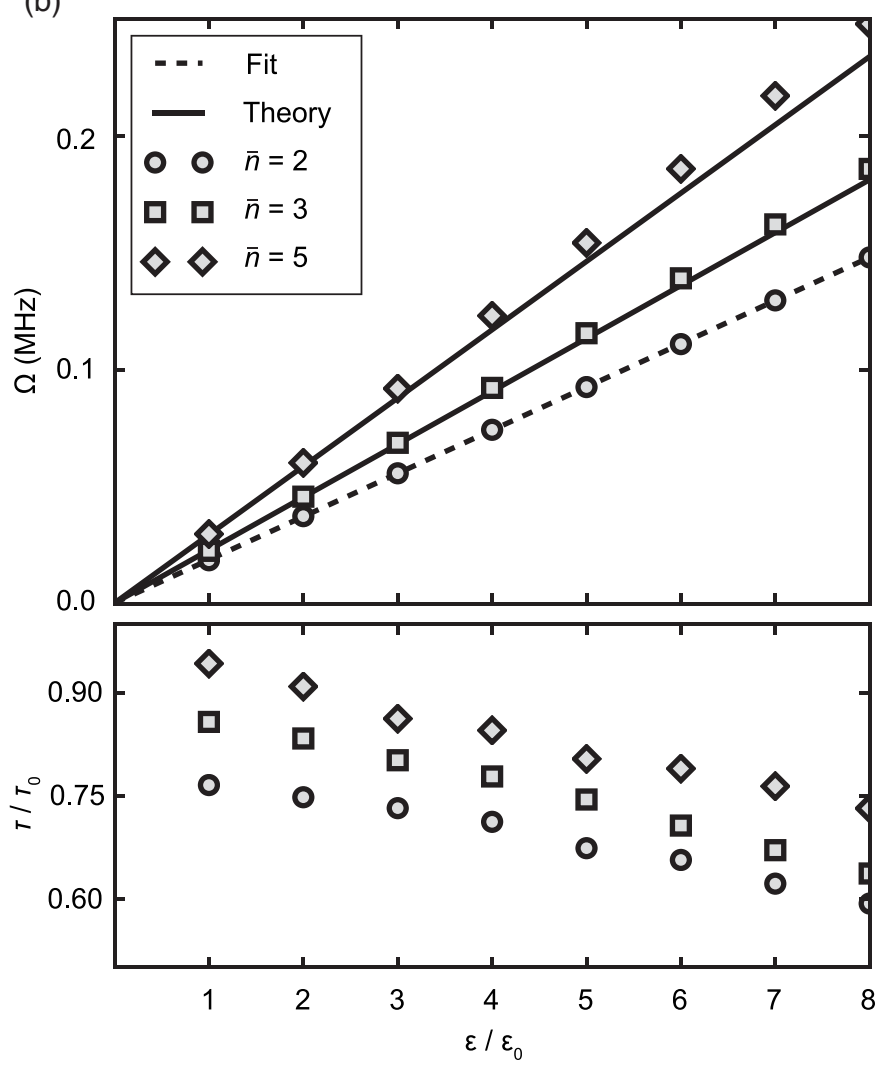

FIG. 3. Characterization of the oscillations. (a) Evolution of the measured parity as a function of time. The initial cat states are even, with $\bar{n}=\left|\alpha_{\infty}\right|^{2}=2,3,5$ (circles, squares, diamonds). The storage drive is either off (black markers) or on (colored markers) with various strengths given in units of a chosen base strength $\epsilon_{0}$. Simulations are shown as solid lines. The minimum of each experimental curve is emphasized for each drive strength (full marker with black contour). (b) A fit of the data gives the frequency $\Omega$ and the time constant $\tau$ of the decaying oscillations. The former is plotted as a function of the relative drive strength $\epsilon / \epsilon_{0}$ (top panel). The case $\bar{n}=2$ is fitted with a linear function (dashed line). Based on this, we make predictions for $\bar{n}=3,5$ (solid lines). The bottom panel shows the characteristic decay time of the oscillations $\tau$, normalized by the decay time of the nondriven case $\tau_{0}$. 
$\Omega=2 \epsilon\left|\alpha_{\infty}\right|$. We chose a first drive strength $\epsilon_{0}$ that gave a single oscillation in parity within the decay time of a Schrödinger cat state with amplitude $\bar{n}=2$. We then repeated the experiment for drive strengths that were multiples of $\epsilon_{0}$ and for different amplitudes of the initial cat states. On each panel the frequency of the oscillations increases with the drive strength. By looking at curves that correspond to the same drive strength over different panels (same color), we see that the frequency of oscillation also increases with the amplitude of the initial state. We obtain theory predictions by numerically integrating the evolution of the density matrix and superimposing them on the data. The parameters of the theory were all provided by the results of independent experiments [26].

We also present in Fig. 3(b) the frequency of the oscillations as a function of the normalized drive strength [Fig. 3(b)]. According to theory, the oscillation frequency should depend linearly on the drive strength. The linear fit for $\bar{n}=2$ gives $\epsilon_{0} / 2 \pi=7 \mathrm{kHz}$. This value, when compared to $\bar{n} \kappa_{2}$, means that we respect the adiabaticity condition for this drive strength. However, when the drive strength increases, this condition is no longer fulfilled. Subsequently, we predict the oscillation frequencies for $\bar{n}=3$ and 5 with good agreement. The difference between the prediction and the data indicates that the stabilized cat state might have had a larger amplitude than the one measured. This is corroborated by the fact that the decoherence time scales for $\bar{n}=3$ and 5 were lower than predicted.

The second panel of Fig. 3(b) shows the evolution of the normalized decay time constant for different cat state amplitudes as a function of the drive strength. If the gate was infinitely slow, the decay constant would be the same as in the nondriven case. However, when $\epsilon / \epsilon_{0}$ increases, the oscillations decay faster. This is explained by the fact that the gate does not perfectly respect the adiabaticity condition $|\epsilon| \ll\left|\alpha_{\infty}\right|^{2} \kappa_{2}$. Nevertheless, when the number of photons in the initial state is larger, the decay constant of the oscillations gets closer to the ideal limit: the adiabaticity condition is easier to fulfill for higher number of photons. Although encoding with a Schrödinger cat state of larger amplitude increases the bit-flip rate, given by $2 \bar{n} \kappa_{1}$, it increases the quality of our manipulation.

Finally, we measured the effect of this protected Rabi rotation on an arbitrary state of the encoding manifold. We represent a state of the protected qubit by a vector in the Bloch sphere. Its coordinates were found by measuring the equivalent Pauli operators for this encoding [29]. The effect of the gate is accurately described by its effect on the 6 cardinal points of an octahedron within the Bloch sphere. We present the results for a manifold encoding using $\left|\alpha_{\infty}\right|^{2}=3$ (Fig. 4). The octahedron on the left shows the initial state. To illustrate the effect of the gate, we chose a specific rotation of $\pi / 2$ using a drive strength $\epsilon=6 \epsilon_{0}$. This corresponds to a gate time of $1.8 \mu \mathrm{s}$. We compared the

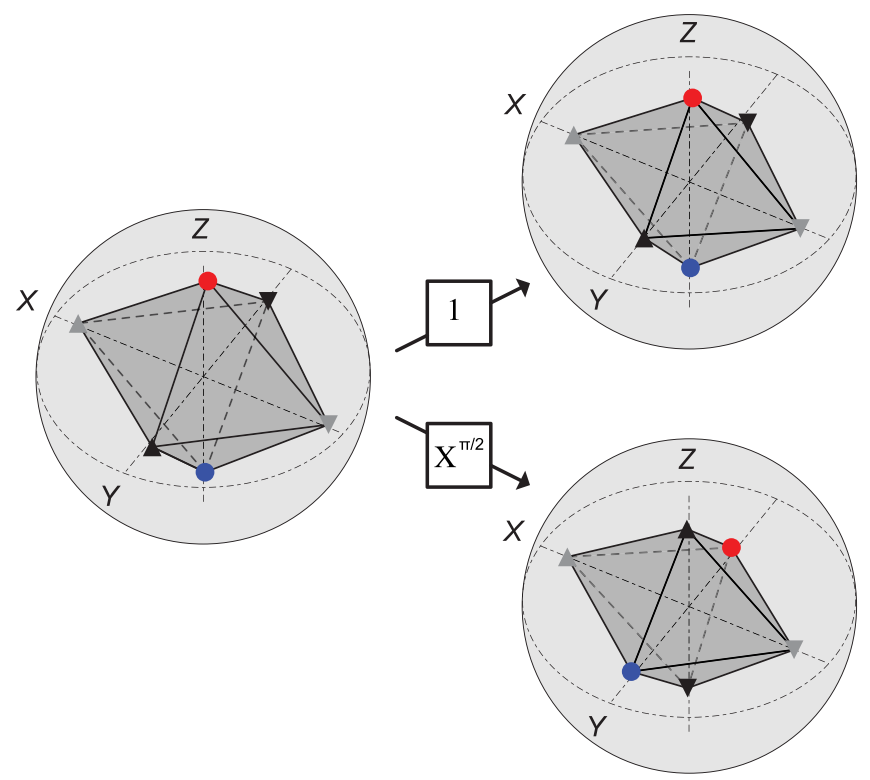

FIG. 4. Gate on cardinal points of the Bloch sphere of the protected manifold. An arbitrary cat state $\mathcal{N}\left[\cos (\theta / 2)\left|\mathcal{C}_{\alpha_{\infty}}^{+}\right\rangle+\sin (\theta / 2) e^{i \phi}\left|\mathcal{C}_{\alpha_{\infty}}^{-}\right\rangle\right]$is represented by a point on a sphere. Six initial states are chosen, corresponding to the cardinal points $(\theta, \phi)=(0,0),(\pi, 0),( \pm \pi / 2,0),(\pi / 2, \pm \pi / 2)$, with $\left|\alpha_{\infty}\right|^{2}=3$, and their equivalent Pauli operators are measured. The markers corresponding to each initial state are, respectively, red and blue circles, gray up or down triangles, and black up or down triangles. The initial octahedron formed by those points (left) is either transformed under the action of the identity (upper right) or a rotation of $\pi / 2$ around the $X$ axis (lower right).

results with those obtained by waiting for the same amount of time without applying a drive, which corresponded to applying the identity. Using the Pauli transfer matrix [30] to represent an operation, we analyze these three processes. We find the fidelities to the respective ideal processes to be $\mathcal{F}=0.87$ for the encoding, 0.81 for the identity, and 0.80 for the $\pi / 2$ rotation [26]. Within these fidelities, we isolate the part that is due to the fidelity of the operation from the part that is due to the quality of the measurement. For this, we normalize the measurement by the contrast of the Wigner function ( 0.841 for $\left|\alpha_{\infty}\right|^{2}=3$ ). We get normalized fidelities of $\mathcal{F}=0.98$ (encoding), 0.90 (identity), and $0.89(\pi / 2$ rotation). The encoding fidelity is slightly smaller than what has been measured in similar implementations [17], likely due to the finite temperature of the reservoir [26]. We see that the fidelities of the rotation and of the identity are similar. This indicates that they are limited by the decoherence of the storage mode. The remaining discrepancy between the rotation and the identity is due to the fact that the gate is not infinitely slow compared to the stabilization, as illustrated in the lower panel of Fig. 3(b).

The next step after manipulating an encoded and protected qubit contained in the stabilized manifold of cat states is to address the fault tolerance of logical operations. A future version of our experiment, which 
should be accessible with current techniques, will increase $\kappa_{2}$ above any coupling to other modes and thus achieve two goals. First, it will improve the gate quality to make it better than a gate on a physical qubit. Second, it will suppress the dephasing due to finite temperature in other modes and thus suppress one remaining decoherence channel. All possible remaining errors would then be equivalent to bit-flip errors, which can be corrected by fault-tolerant joint parity measurements $[31,32]$ on several cavities.

We acknowledge Victor Albert and Liang Jiang for helpful discussions, Kyle Serniak and Luke Burkhart for their work on the fabrication process, and Rémi Bisognin and Renan Goupil for their participation in the experiment. Facilities use was supported by the Yale SEAS clean room, YINQE, and NSF MRSEC DMR-1119826. This research was supported by the Army Research Office (ARO) under Grant No. W911NF-14-1-0011. J. B. and K. C. acknowledge partial support from the ARO Grant No. W911NF-161-0349. P. R. acknowledges partial support from the Air Force Office Scientific Research under Grant No. FA955015-1-0015. S. S. acknowledges partial support from the ARO Grant No. W911NF-14-1-0563. C. A. acknowledges support from the NSF Graduate Research Fellowship under Grant No. DGE-1122492.

[1] B. Misra and E. C. G. Sudarshan, Zeno's Paradox in Quantum Theory, J. Math. Phys. (N.Y.) 18, 756 (1977).

[2] W. M. Itano, D. J. Heinzen, J. J. Bollinger, and D. J. Wineland, Quantum Zeno Effect, Phys. Rev. A 41, 2295 (1990).

[3] S. R. Wilkinson, C. F. Bharucha, M. C. Fischer, K. W. Madison, P. R. Morrow, Q. Niu, B. Sundaram, and M. G. Raizen, Experimental Evidence for Non-Exponential Decay in Quantum Tunnelling, Nature (London) 387, 575 (1997).

[4] P. Facchi, V. Gorini, G. Marmo, S. Pascazio, and E. C. G. Sudarshan, Quantum Zeno Dynamics, Phys. Lett. A 275, 12 (2000).

[5] A. Beige, D. Braun, B. Tregenna, and P. L. Knight, Quantum Computing Using Dissipation to Remain in a Decoherence-Free Subspace, Phys. Rev. Lett. 85, 1762 (2000).

[6] A. Carollo, M. F. Santos, and V. Vedral, Coherent Quantum Evolution via Reservoir Driven Holonomies, Phys. Rev. Lett. 96, 020403 (2006).

[7] O. Oreshkov and J. Calsamiglia, Adiabatic Markovian Dynamics, Phys. Rev. Lett. 105, 050503 (2010).

[8] P. Zanardi and L. C. Venuti, Coherent Quantum Dynamics in Steady-State Manifolds of Strongly Dissipative Systems, Phys. Rev. Lett. 113, 240406 (2014).

[9] V. V. Albert, B. Bradlyn, M. Fraas, and L. Jiang, Geometry and Response of Lindbladians, Phys. Rev. X 6, 041031 (2016).

[10] R. Azouit, F. Chittaro, A. Sarlette, and P. Rouchon, Towards Generic Adiabatic Elimination for Bipartite Open Quantum Systems, Quantum Sci. Technol. 2, 044011 (2017).
[11] M. Wolinsky and H. J. Carmichael, Quantum Noise in the Parametric Oscillator-From Squeezed States to CoherentState Superpositions, Phys. Rev. Lett. 60, 1836 (1988).

[12] M. Mirrahimi, Z. Leghtas, V. V. Albert, S. Touzard, R. J. Schoelkopf, L. Jiang, and M. H. Devoret, Dynamically Protected Cat-Qubits: A New Paradigm for Universal Quantum Computation, New J. Phys. 16, 045014 (2014).

[13] Z. Leghtas, S. Touzard, I. M. Pop, A. Kou, B. Vlastakis, A. Petrenko, K. M. Sliwa, A. Narla, S. Shankar, M. J. Hatridge, M. Reagor, L. Frunzio, R. J. Schoelkopf, M. Mirrahimi, and M. H. Devoret, Confining the State of Light to a Quantum Manifold by Engineered Two-Photon Loss, Science 347, 853 (2015).

[14] L. G. Lutterbach and L. Davidovich, Method for Direct Measurement of the Wigner Function in Cavity QED and Ion Traps, Phys. Rev. Lett. 78, 2547 (1997).

[15] L. Sun, A. Petrenko, Z. Leghtas, B. Vlastakis, G. Kirchmair, K. M. Sliwa, A. Narla, M. Hatridge, S. Shankar, J. Blumoff, L. Frunzio, M. Mirrahimi, M. H. Devoret, and R. J. Schoelkopf, Tracking Photon Jumps with Repeated Quantum Non-Demolition Parity Measurements, Nature (London) 511, 444 (2014).

[16] N. Ofek, A. Petrenko, R. Heeres, P. Reinhold, Z. Leghtas, B. Vlastakis, Y. H. Liu, L. Frunzio, S. M. Girvin, L. Jiang, M. Mirrahimi, M. H. Devoret, and R. J. Schoelkopf, Extending the Lifetime of a Quantum Bit with Error Correction in Superconducting Circuits, Nature (London) 536, 441 (2016).

[17] R. W. Heeres, P. Reinhold, N. Ofek, L. Frunzio, L. Jiang, M. H. Devoret, and R. J. Schoelkopf, Implementing a Universal Gate Set on a Logical Qubit Encoded in an Oscillator, Nat. Commun. 8, 94 (2017).

[18] P. Facchi and S. Pascazio, Quantum Zeno Subspaces, Phys. Rev. Lett. 89, 080401 (2002).

[19] J. M. Raimond, P. Facchi, B. Peaudecerf, S. Pascazio, C. Sayrin, I. Dotsenko, S. Gleyzes, M. Brune, and S. Haroche, Quantum Zeno Dynamics of a Field in a Cavity, Phys. Rev. A 86, 032120 (2012).

[20] F. Schafer, I. Herrera, S. Cherukattil, C. Lovecchio, F. S. Cataliotti, F. Caruso, and A. Smerzi, Experimental Realization of Quantum Zeno Dynamics, Nat. Commun. 5, 3194 (2014).

[21] A. Signoles, A. Facon, D. Grosso, I. Dotsenko, S. Haroche, J. M. Raimond, M. Brune, and S. Gleyzes, Confined Quantum Zeno Dynamics of a Watched Atomic Arrow, Nat. Phys. 10, 715 (2014).

[22] L. Bretheau, P. Campagne-Ibarcq, E. Flurin, F. Mallet, and B. Huard, Quantum Dynamics of an Electromagnetic Mode that Cannot Contain N Photons, Science 348, 776 (2015).

[23] M. Reagor, H. Paik, G. Catelani, L. Y. Sun, C. Axline, E. Holland, I. M. Pop, N. A. Masluk, T. Brecht, L. Frunzio, M. H. Devoret, L. Glazman, and R. J. Schoelkopf, Reaching $10 \mathrm{~ms}$ Single Photon Lifetimes for Superconducting Aluminum Cavities, Appl. Phys. Lett. 102, 192604 (2013).

[24] S. Haroche and J. M. Raimond, Exploring the Quantum: Atoms, Cavities and Photons. (Oxford University Press, New York, 2006).

[25] C. Axline, M. Reagor, R. Heeres, P. Reinhold, C. Wang, K. Shain, W. Pfaff, Y. Chu, L. Frunzio, and R. J. Schoelkopf, 
An Architecture for Integrating Planar and 3D cQED Devices, Appl. Phys. Lett. 109, 042601 (2016).

[26] See Supplemental Material at http://link.aps.org/ supplemental/10.1103/PhysRevX.8.021005 for details.

[27] P. Bertet, A. Auffeves, P. Maioli, S. Osnaghi, T. Meunier, M. Brune, J. M. Raimond, and S. Haroche, Direct Measurement of the Wigner Function of a One-Photon Fock State in a Cavity, Phys. Rev. Lett. 89, 200402 (2002).

[28] B. Yurke and D. Stoler, Generating Quantum-Mechanical Superpositions of Macroscopically Distinguishable States via Amplitude Dispersion, Phys. Rev. Lett. 57, 13 (1986).

[29] B. Vlastakis, A. Petrenko, N. Ofek, L. Y. Sun, Z. Leghtas, K. Sliwa, Y. H. Liu, M. Hatridge, J. Blumoff, L. Frunzio, M. Mirrahimi, L. Jiang, M. H. Devoret, and R. J. Schoelkopf, Characterizing Entanglement of an Artificial Atom and a Cavity Cat State with Bell's Inequality, Nat. Commun. 6, 8970 (2015).

[30] J. M. Chow, J. M. Gambetta, A. D. Córcoles, S. T. Merkel, J. A. Smolin, C. Rigetti, S. Poletto, G. A. Keefe, M. B. Rothwell, J. R. Rozen, M. B. Ketchen, and M. Steffen, Universal Quantum Gate Set Approaching Fault-Tolerant Thresholds with Superconducting Qubits, Phys. Rev. Lett. 109, 060501 (2012).

[31] J. Cohen, W. C. Smith, M. H. Devoret, and M. Mirrahimi, Degeneracy-Preserving Quantum Nondemolition Measurement of Parity-Type Observables for Cat Qubits, Phys. Rev. Lett. 119, 060503 (2017).

[32] C. Wang, Y. Y. Gao, P. Reinhold, R. W. Heeres, N. Ofek, K. Chou, C. Axline, M. Reagor, J. Blumoff, K. M. Sliwa, L. Frunzio, S. M. Girvin, L. Jiang, M. Mirrahimi, M. H. Devoret, and R. J. Schoelkopf, A Schrodinger Cat Living in Two Boxes, Science 352, 1087 (2016). 